\title{
Erhöhtes Schlaganfallrisiko bei Diabetes muss nicht sein
}

Bei guter Blutdruckkontrolle ist das Schlaganfallrisiko von Typ-2-Diabetikern nicht höher als das von durchschnittlichen Nichtdiabetikern. Darauf deutet eine große Registerstudie aus Schweden hin.

Menschen mit Typ-2-Diabetes haben ein 1,5- bis 3-mal so hohes Risiko für einen Schlaganfall wie Nichtdiabetiker - sofern man sie als Gruppe betrachtet. Differenziert man die Diabetiker nach der Höhe ihres Blutdrucks, ergibt sich laut einer prospektiven Studie aus Schweden ein etwas anderes Bild. Das erhöhte Schlaganfallrisiko betrifft dann nur Patienten mit Werten ab 130/80 mmHg. Bei niedrigeren Drücken unterscheidet sich das Schlaganfallrisiko dagegen nicht vom Bevölkerungsdurchschnitt ohne Diabetes.

\section{Risiko um 43 \% höher als in der Bevölkerung}

Für die Studie wurden über 400.000 erwachsene Typ-2-Diabetiker aus dem nationalen Diabetesregister mit einer Bevölkerungsstichprobe von 1,9 Millionen Schweden mit gleichem Alter und Geschlecht, aber ohne Diabetes verglichen. In der Beobachtungszeit von median vier Jahren wurde bei 19.548 Diabetikern $(4,8 \%)$ und 61.690 Nichtdiabetikern (3,2\%) ein erster Schlaganfall diagnostiziert. Das entspricht Ereignisraten von 10,63 bzw. 6,80 pro 1000 Personenjahre. Das adjustierte Schlaganfallrisiko lag damit in der Diabetikergruppe um $43 \%$ höher als in der Kontrollgruppe.

\section{Minus bei hämorrhagischen Insulten}

Das Zusatzrisiko bestand ab einem Blutdruck von 130/80 $\mathrm{mmHg}$. Es steigerte sich druckabhängig von $20 \%$ bei Werten von 130-139/80-89 mmHg bis auf $97 \%$ bei Werten ab 160/100 mmHg. Für einen ischämischen Schlaganfall war das Risiko in der Gesamtgruppe der Diabetiker um 48\% erhöht. Ein Risikoanstieg war hier schon ab einem Blutdruck von 110-119/65-69 mmHg zu erkennen. Bei Werten ab 160/100 mmHg war das Risiko gut doppelt so hoch wie bei den Nichtdiabetikern.

Ein hämorrhagischer Schlaganfall trat bei den Diabetikern insgesamt nur um $8 \%$ häufiger auf als in der Vergleichsgruppe. In den Blutdruckkategorien 120-129/70-79 mmHg und 130139/80-89 mmHg war das Risiko sogar signifikant niedriger als bei den Nichtdiabetikern, um $34 \%$ und $13 \%$. Bei normalem Blutdruck wird das Exzessrisio für einen ischämischen Schlaganfall danach durch das verminderte Risiko eines hämorrhagischen Schlaganfalls aufgewogen.

Laut den Studienautoren verdeutlichen die Ergebnisse die Wichtigkeit einer guten Blutdruckeinstellung: „Menschen mit Typ-2-Diabetes können, wenn ihr Blutdruck gut kontrolliert ist, ein Schlaganfallrisiko haben, das ähnlich ist wie das der Allgemeinbevölkerung“, so das Fazit der Ärzte um Christina Hedén Ståhl von der Universität Göteborg. Ein Manko der Studie besteht allerdings darin, dass für die Kontrollgruppe keine Angaben zum Blutdruck vorlagen. Ein Vergleich von Diabetikern und
Nichtdiabetikern derselben Druckkategorien war daher nicht möglich.

Dr. Beate Schumacher

Quelle: Hedén Ståhl C et al. Long-term excess risk of stroke in people with

Type 2 diabetes in Sweden according to blood pressure level. Diabet Med 2017

\section{Blutzuckermessung im Schweiß}

\section{Hoffnung für Diabetiker}

Wissenschaftler haben einen Sensor entwickelt, der den Blutzuckerspiegel anhand von Hautschweiß misst.

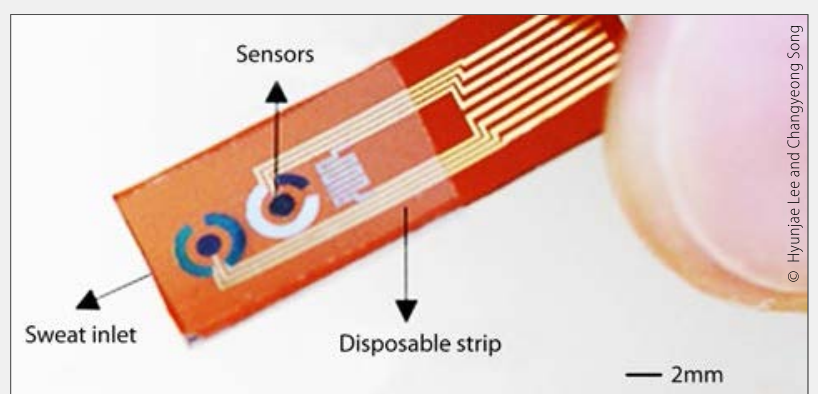

Einen Sensor zur Blutzuckermessung in Schweiß haben Wissenschaftler aus Südkorea entwickelt.

Bereits eine Millionstel Liter Schweiß soll ausreichen, um den Blutzuckerspiegel zu messen. Dazu haben südkoreanische Wissenschaftler einen Sensor entwickelt, wie BBC online unter Berufung auf das Fachmagazin "Science Advances" berichtet.

In Versuchen mit Mäusen koppelten die Forscher der Seoul National University den Schweißsensor außerdem mit dünnen Spritznadeln, um eine Diabetes-Arznei (Metformin) automatisch angepasst an den Blutzuckerspiegel zu verabreichen. Der Sensor passe sich an die Haut an und bleibe somit auch bei Bewegungen haften. Er messe so exakt, dass möglicherweise auch Diabetes-Patienten davon profitieren könnten, so die BBC. Erste Tests mit Menschen vor und nach dem Essen hätten gezeigt, dass die Ergebnisse der Schweißmessung mit denen traditioneller Geräte "gut übereinstimmen“, so die BBC. Im nächsten Schritt sollen die Sensoren einem Langzeittest unterzogen werden. Noch bleiben nämlich eine Reihe von Herausforderungen. Die Messung im Schweiß gestalte sich schwer, da dieser weniger Zucker beinhalte als Blut. Zudem verfälschten andere im Schweiß vorhandene Substanzen wie Milchsäure die Messresultate. Das Messgerät beinhalte daher mehrere Sensoren: drei zur Beobachtung des Blutzuckerspiegels, vier zur Messung des pH-Werts und zudem einen Feuchtigkeitssensor, um die Schweißmenge zu analysieren.

Bislang müssen Diabetiker ihren Blutzuckerspiegel durch regelmäßiges Pieksen kontrollieren. Allerdings gibt es mit der Flash-Glucose-Messung bereits eine schmerzarme Alternative, die in Deutschland seit Anfang 2017 von mehreren Krankenkassen erstattet wird. $e b$ 